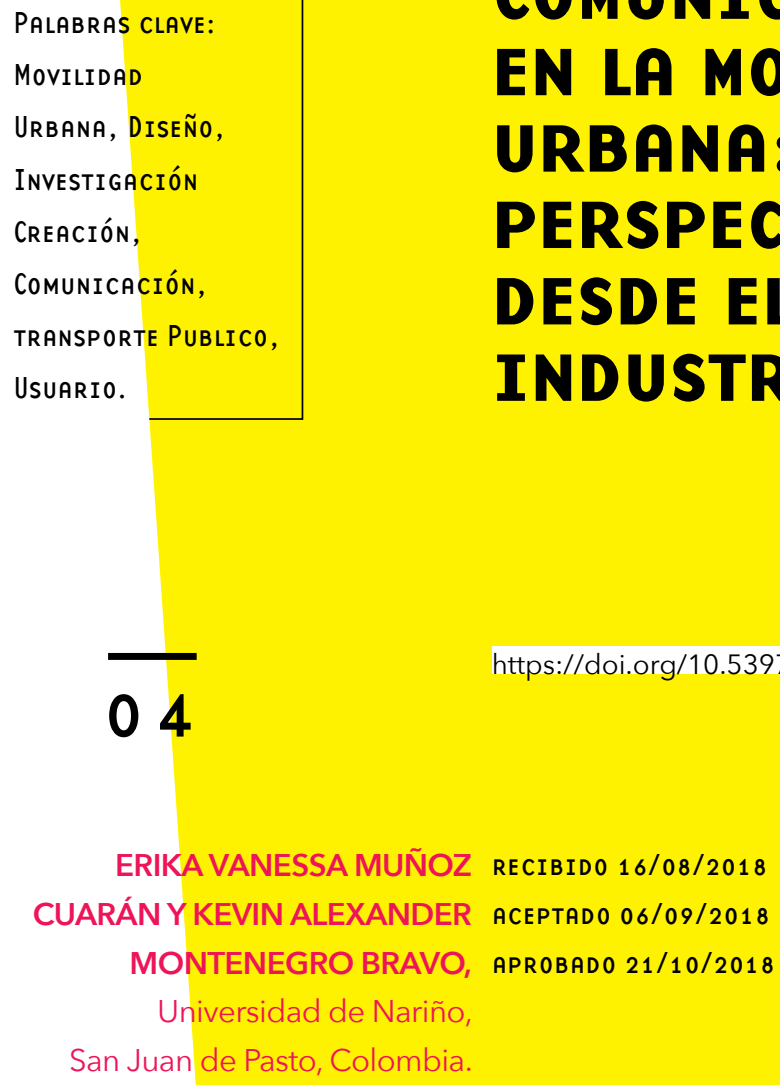

04

ERIKA VANESSA MUÑOZ RECIBIDO 16/08/2018

CUARÁN Y KEVIN ALEXANDER ACEPTADO 06/09/2018

MONTENEGRO BRAVO, APROBADO 21/10/2018

Universidad de Nariño,

San Juan de Pasto, Colombia.

https://doi.org/10.53972/RAD.eifd.2018.1.7

Resumen: La ciudad de San Juan de Pasto está siempre en constante cambio cultural, económico y en mayor medida de infraestructura, por lo que se generan múltiples problemáticas en el entorno, una de estas más específicamente es la comunicación en el transporte público, por lo tanto no se han generado suficientes soluciones eficientes para la ayuda de la población con esta problemática, el proyecto de "COMUNICACIÓN EN LA MOVILIDAD: nuevas perspectivas desde el diseño industrial" busca generar soluciones optimas y más importante, poniendo como punto inicial una disciplina propia de ello y al mismo tiempo integrar múltiples disciplinas que se apoyen unas a otras para hacer un trabajo más sólido, para lograr este propósito se aplica una estrategia metodológica desde la investigación creación. Se caracteriza por desarrollar procesos altamente diversos, influenciados por el carácter personal y sus experiencias en el contexto y lenguaje formal estético. Se adquieren conocimientos utilizando diferentes herramientas fuera de las disciplinas creativas, las cuales se mezclan según la intención del proyecto con habilidades desarrolladas anteriormente desde el transcurso de su aprendizaje en la carrera, sensibilizándose con el medio que habita; este nuevo conocimiento se da en esa capacidad de mover fronteras entre lo que existe y lo que no. 


\section{La comunicación en la movilidad urbana ha} pasado por diferentes bases y evoluciones, inclinadas más al ámbito visual. A partir de esto, según el estudio de avante SETP que es el encargado actual del sistema integrado de transporte, ha logrado implementar el proyecto de movilidad en la ciudad de San Juan de Pasto, Nariño, en el que se centran diferentes puntos para generar una correcta movilidad en la ciudad.

La parte visual de señalética en los paraderos fue creada y esta implementada desde el 28 de junio del año 2017. (AVANTE, 2017) se pretende incentivar a la población a utilizar este tipo de paradas para acenso y descenso de pasajeros, no obstante cabe recalcar que las paradas no cuentan con un sistema para diferentes tipos de usuarios (niños, ancianos, personas con discapacidad) ya que estas paradas no tienen las condiciones mínimas de comodidad para que una persona tome el transporte público, la falta de investigación, exploración y gestión por parte de AVANTE en cuanto a las múltiples disciplinas que se pueden acoplar a este tipo de proyectos es la parte principal de un problema, porque no se ofrece un diseño adecuado a la población para incentivar el uso de este, no existe un elemento que facilite a la persona a tomar el autobús y que tenga unas condiciones de protección climática para esto, por otra parte las paradas actuales están ubicadas cada cierta distancia una de otra y no tienen en cuenta los puntos de reunión o concentración de personas más recurrentes en la ciudad, información escasa y mal distribuida es otro problema generado por estas paradas, todo esto son múltiples problemáticas que van ligadas unas con otras por lo que la intervención del diseño industrial puede ser un aspecto positivo para la generación de nuevos espacios y la interacción con estos, incluyendo cierta parte de la investigación creación como lo es la experiencia del diario vivir de las personas con relación a este tipo de elementos. 


\section{Metodología}

\section{Investigación Creación Conocimiento del Contexto | Disparador | Materialización}

La investigación creación se caracteriza por desarrollar procesos altamente diversos, procesos influenciados por el carácter personal del creador, su contexto y lenguaje formal estético. Por lo tanto, no es prudente encajar dichos procesos en métodos estándar o rígidos que limiten en cierta manera la creatividad. El investigador-creador generalmente utiliza diversas herramientas fuera de las disciplinas creativas, las cuales combina según la intención del proyecto, sus propias habilidades y experiencia. (Ballesteros, 2018)

En ese sentido, no es posible afirmar que la investigación creación se rige bajo un solo orden o método, pero si deben existir ciertos requerimientos necesarios para generar conocimiento desde un modelo de investigación creación. Se plantean entonces tres componentes de una estructura general para el proceso de investigación creación, que pueden ser secuenciales, simultáneos o en orden indistinto.

\section{Contextualización}

Consiste en conocer y apropiar la realidad para alimentar los procesos de creación. Se equipara a una búsqueda de diversos tipos de información, lo cual determina la originalidad y novedad de la producción de conocimiento mediante la revisión de referentes desde su marco disciplinar. El investigador creador apropia información de tal manera que esta proporciona una contribución significativa a sus intenciones de conocimiento.

\section{Actividades:}

- Recolección de información respecto a las estrategias de generar una movilidad segura e integral en eventos desarrollados por la alcaldía y AVANTE en la región.

- Recopilación de productos desarrollados en la zona urbana que manifiesten una comunicación adecuada para el peatón sobre el transporte público a nivel regional.

- Consulta respecto a referentes nacionales e internacionales relacionados con el desarrollo de paraderos de transporte público creativos vinculados a la movilidad como casos de éxito. 


\section{Sensación detonante}

Consiste en la toma de decisiones respecto a la interpretación, reflexión y crítica respecto a la información del contexto recabada. Así, el investigador creador asume una postura frente a la realidad contextual que le permite enfatizar en un aspecto particular de su interés. En esta etapa se detona la concreción en términos de formalización, pues el creador encuentra la intención de su creación.

Actividades:

- Análisis de diseño sobre los productos previamente desarrollados en la movilidad que permita dar una valoración formal estética y práctico funcional.

- Análisis de la estructura organizativa y del factor diferenciador en los casos de éxito identificados como emprendimientos creativos desde eventos culturales.

- Desarrollo de propuestas conceptuales para la generación de productos.

\section{Conformación plástica}

Consiste en la transformación de la realidad implícita en la creación, la cual adquiere una forma y lenguaje específicos que le permiten comunicar el mensaje que contiene. En otras palabras, se concreta la creación en un producto plástico sensorial.

\section{Actividades:}

- Determinación de requerimientos de diseño.

- Desarrollo de propuestas.

- Bocetación.

- Elaboración de modelos formales-funcionales.

- Selección de alternativas.

- Desarrollo de alternativa final (prototipos).

- Comprobación y ajustes.

- Elaboración de producción piloto.

En cada una de las etapas anteriormente descritas se aplicarán, según criterio del investigador creador, las herramientas que su racionalidad basada en la experiencia le permitan de manera espontánea orientar su proceso creativo desde esta perspectiva investigativa. 


\section{Mapa Metodológico Investigación creación}

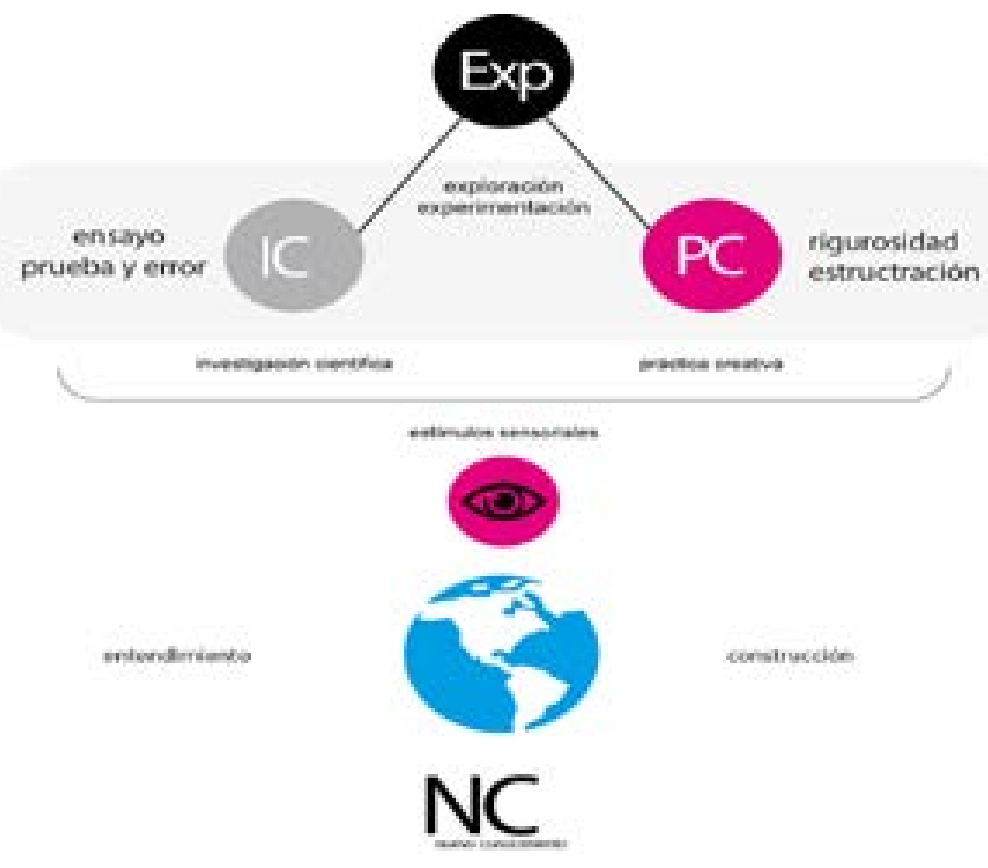

Figura 1. Investigar creando autor Danilo Calvache

Esta gráfica ayuda a comprender la metodología en una serie de pasos para entender la problemática que se está abordando, es así como el diseñador industrial conceptualiza los procesos diversos que contiene una investigación, principalmente se cuenta con la exploración o contextualización del medio, en este aspecto los estímulos sensoriales juegan un papel importante en cómo puedo ver el mundo y desde qué perspectiva veo los acontecimientos que pasan alrededor, una vez que se entienda el porqué de las cosas, se pasa a la construcción de un nuevo conocimiento tomando varios puntos de vista desde otras disciplinas, este nuevo conocimiento está implícito en productos o servicios que son las soluciones que aquejan dicho problema.

El siguiente mapa mental que se construyó relata el proceso que hemos venido llevando acabo con la recolección de información y observación del contexto, cabe recalcar que este proyecto no está finalizado ya que hemos tenido rigurosidad en extraer toda la información posible para tomar decisiones que ayuden a enriquecer el proyecto. 


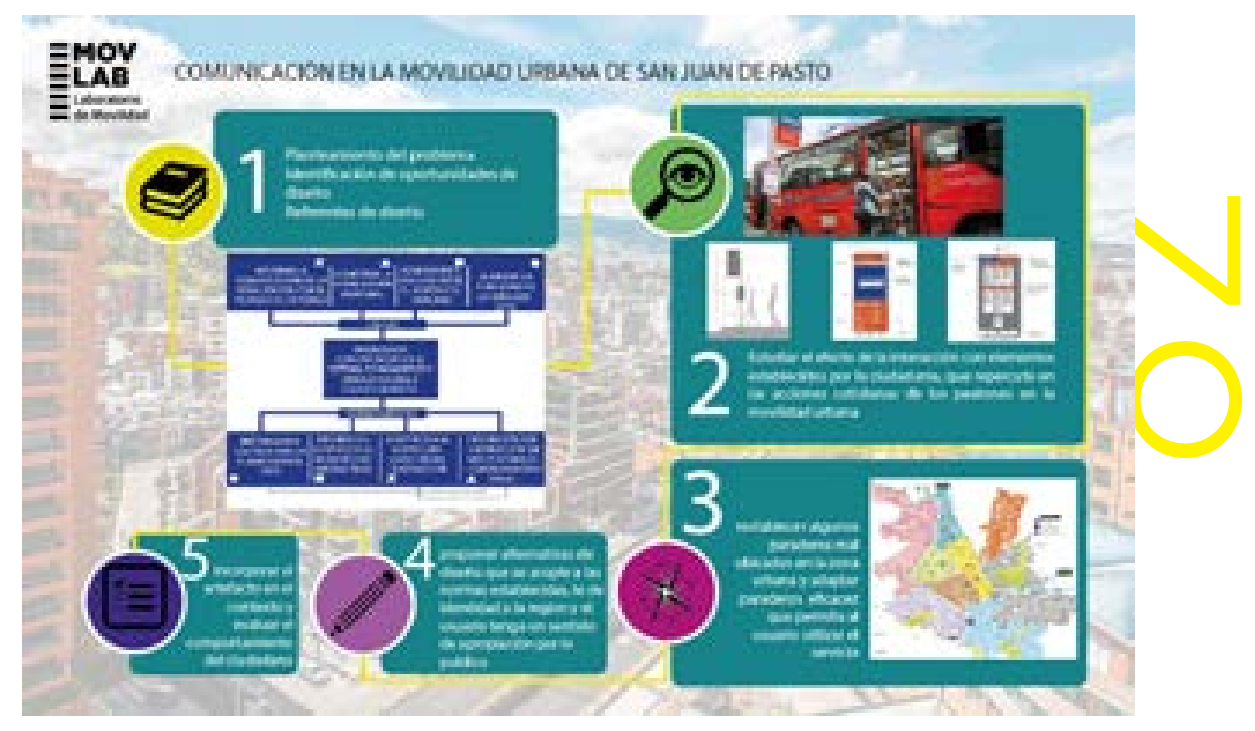

Figura 2. Infografía metodológica de los pasos básicos

Se trazó un mapa mental básico de los factores de investigación que intervenían en el fenómeno de la movilidad urbana el cual sirviera para recolectar la mayor información posible de las problemáticas claves para desarrollar una solución o un producto que impactara de manera positiva en el aspecto de comunicación en la movilidad urbana en la ciudad de Pasto.

\section{EXPLORACIÓN:}

La exploración en este caso fue detallar ciertos comportamientos que el ciudadano maneja en la ciudad y cómo se desenvuelve al enfrentar tráfico vehicular en las zonas mayormente concurridas, así mismo se pudo dar cuenta que el ser humano está en constante movimiento y tiene la capacidad de buscar soluciones ante las situaciones de caos, en la gráfica se puede observar que el transeúnte del transporte público desciende del vehículo para poder caminar hacia su lugar de destino, pero ¿por qué se da esto? Tal vez si vemos más allá, hay factores detonantes como lo es buscar otros medios de transporte que no afecten al medio ambiente y que generen una mayor circulación, así mismo la ciudad y entes gubernamentales deben responder ante estos problemas, con una mejor infraestructura, pedagogía al ciudadano, medios de transportes alternativos, etc. En segundo lugar, al no existir una concentración de personas en un paradero, los medios de transporte urbanos se ven en la forzada 


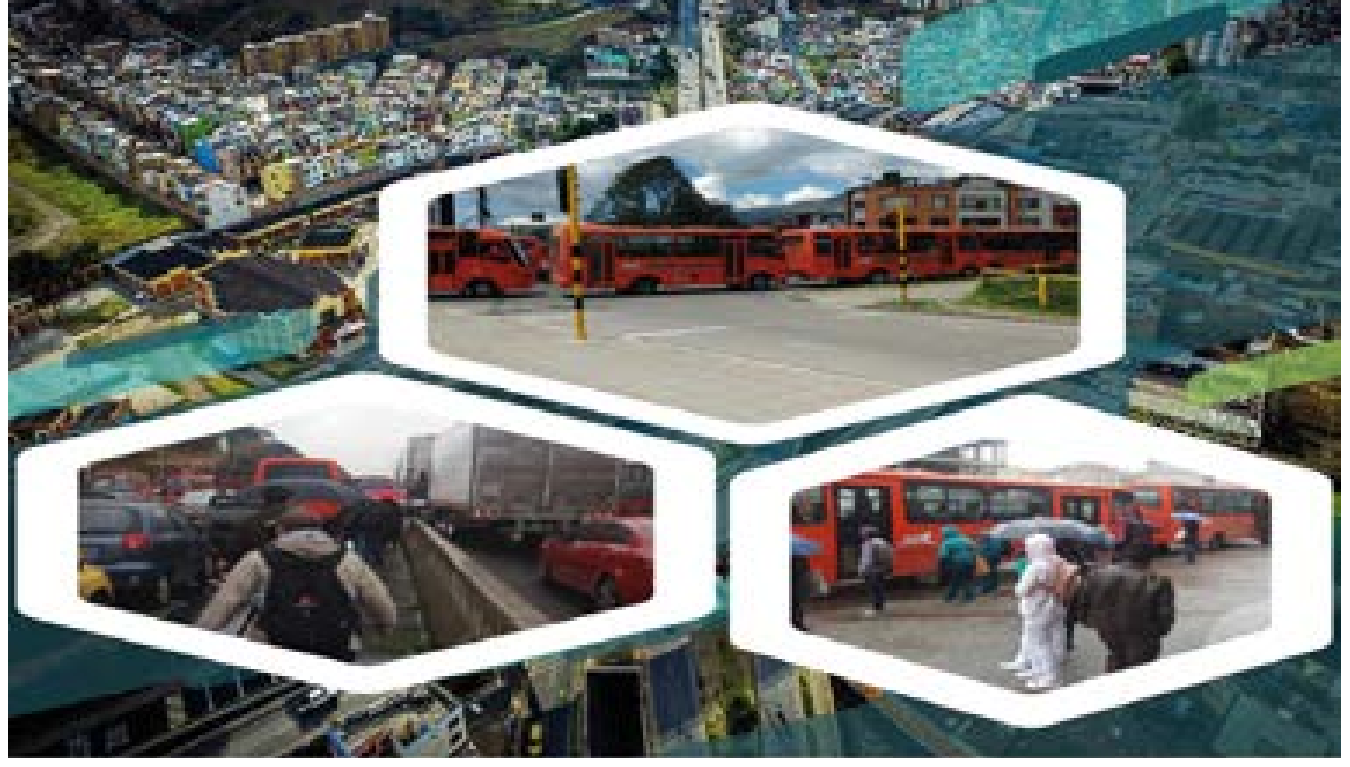

obligación de recogerlos donde las personas estén situadas, perjudicando los tiempos y congestionando las vías, pero es un mal necesario ya que si no lo hacen el sistema de transporte tendría pérdidas económicas.

"La dirección de transporte publico metropolitano de chile define como "punto de parada" al espacio físico de conexión entre usuarios y los servicios de buses del sistema de transportes" (Accesible, 2018)

El punto de parada puede tener diferentes elementos, lo que dependerá de cada situación en función de la intensidad de uso, el flujo de peatones en la vía pública que conecta con el paradero y el espacio disponible.

Todo paradero debe contar como mínimo con una señal de parada e idealmente un asiento. La señal permite al usuario informarse de los servicios que se detienen en esa ubicación. Un paradero puede contar o no con un refugio, la existencia dependerá en gran medida del espacio disponible en la acera. El paradero debe emplearse, siempre de ahí la importancia que la ciudad tenga estos espacios.

\section{RESULTADOS Y/O PRODUCTOS ESPERADOS}

Los artefactos diseñados para el entorno urbano deben cumplir con ciertos requerimientos esenciales en cuanto a durabilidad, usabilidad, funcionalidad, legibilidad, comodidad, además de ser un atractivo perceptual estético debe proporcionar al ciudadano una interacción con el ambiente facilitando las actividades cotidianas, de ahí su importancia del diseño como investigación - creación que deben estar sujetas al estudio del ser humano en contexto determinado. 
Se espera con estos objetos en los espacios urbanos (el comercio, instituciones bancarias, entidades gubernamentales y la misma ciudadanía) sean beneficiados por la dinámica de una ciudad estructurada, desde sus sistemas de circulación, estaciones, paraderos y puestos de información (MUPIS) propuesta generada desde la imagen de la ciudad con estudios de su origen, su historia, su creación, que den a los ciudadanos una manera más óptima, sencilla y agradable de tomar un transporte público y a su vez crear una identidad cultural.

Se espera un reconocimiento a nivel nacional e internacional, así como participación en eventos de proyectos de movilidad, la elaboración e implementación del proyecto en la ciudad de pasto y dar a conocer la disciplina del diseño industrial como un ente importante en el desarrollo de productos y proyectos que ayuden a un mejor desarrollo social, económico y cultural.

\section{Conclusión}

COMUNICACIÓN EN LA MOVILIDAD URBANA: NUEVAS PERSPECTIVAS DESDE EL DISEÑO INDUSTRIAL

- En relación a lo antes expuesto, podemos decir que se han cumplido un número de pasos importantes con relación a la identificación de problemas en el transporte público de la ciudad de San Juan de Pasto, consta resaltar que este trabajo de investigación-creación no está en su punto más alto de concluir ya que faltan muchos factores y pasos del cronograma, a pesar de esto se puede decir que se ha hecho un avance muy importante y conforme a la investigación que se ha desarrollado por el momento, esta investigación sirvió para interiorizarnos más en el tema y aprender de una manera más adecuada sobre las problemáticas urbanas, al haber expuesto a la comunidad académica acerca del proyecto fue muy importante ya que se identificaron y se reunieron diferentes puntos de vista tanto interdisciplinarios como de la misma línea de diseño pero en diferentes entornos, con esto se va perfeccionando y aprendiendo la nueva metodología de investigación-creación la cual es una herramienta muy útil y poco conocida, darle la importancia que se merece esta metodología nos acerca más un 
paso en la evolución e implementación de nuevas técnicas de la investigación en el diseño.

- Lo que continua de este proyecto es evaluar, clasificar e implementar la información recaudada durante este tiempo, cabe resaltar que gracias al encuentro de semilleros conocimos algunos expertos en múltiples temas y se obtuvo una grata realimentación para poder continuar con el proyecto, lo que se espera en un futuro es generar soluciones óptimas para el buen uso de los paraderos y del transporte público, y generar nuevos conocimientos que sirvan para solucionar futuros fenómenos creados en torno a la movilidad

Como su nombre lo indica, la conclusión de un proyecto es la parte final de cualquier trabajo de investigación, en ésta el investigador sintetiza los resultados de su investigación, producto del alcance de los objetivos generales y específicos trazados inicialmente.

\section{Referencias}

AVANTE. (JUNIO de 2017). Obtenido de www.avante.gov.co.: https:// www.avante.gov.co/operaciones/senaletica-y-paraderos

Ballesteros, M. (2018). INVESTIGAR CREANDO? una guia para la investigaciòn-creaciòn en la academia. Bogota: UNIVERSIDAD DEL BOSQUE.

Accesible, c. c. (Marzo de 2018). Ciudad accesible. Obtenido de http://www.ciudadaccesible.cl/wp-content/uploads/2018/03/ Ficha-14-Paraderos-y-Refugios-Peatonales.pdf

Biblioteca Departamental del Valle. (2014). Recuperado el 3 de octubre de 2016, de http://www.bibliovalle.gov.co/publicaciones. php/publicacion/titulo/Sala-Hellen-Keller

Real Academia Española. (2016). Real Academia Española. Obtenido de http://dle.rae.es/?id=A58xn3c

Significados. (s.f.). Significados. Obtenido de https://www.significados. com/sinestesia/

Alcaldía de Santiago de Cali. (28 de febrero de 2012). Recuperado el 07 de septiembre de 2016, de Alcaldía de Cali: http://www.cali. gov.co/publicaciones/por_una_mayor_inclusin_de_las_personas_ con_discapacidad_pub

El Diario. (22 de 06 de 2018). La discapacidad no puede ser un 
impedimiento laboral. El Diario, págs. 1-2.

Rodríguez Ferro , F. E. (2011). Documento de soporte conceptual, metodológico y práctico sobre. Bogotá.

Comité sobre los Derechos de las Personas con Discapacidad. .

(2016). Observaciones finales sobre el informe inicial de Colombia. Bogotá: Naciones Unidas.

Convención de las personas en condición de discapacidad. (2006).

Convención Internacional sobre los Derechos de las Personas con

Discapacidad. New York: Naciones Unidas.

discapacidad, G. d. (2014). Informe regional sobre la medición de la discapacidad. Santiago: CEPAL.

Collado, M. H. (2013). Situación Mundial de la Discapacidad.

Tegucigalpa: Universidad Ncional Autónoma de Honduras.

Banco De Desarrollo De América Latina CAF. (2011). Desarrollo

urbano y movilidad en América Latina. Obtenido de www.caf.com:

http://scioteca.caf.com/handle/123456789/419

Barranquilla, A. D. (2018). Alcaldía De Barranquilla. Obtenido de

http://www.barranquilla.gov.co/planmaestromovilidad/index.php/

que-es-el-pmm\#.WsUTNIjOXIU

CEPAL, C. E. (2008). Anuario estadistico De América Latina y el Caribe.

Chile : Naciones Unidadas.

Sobre los autores
Erika Vanessa Muñoz Cuaran, estudiante del

Departamento de Diseño Industrial de la

Universidad de Nariño de la ciudad de San

Juan de Pasto. Nací en la ciudad de Pasto

en Abril 17 de 1992 realice mis estudios de

diseño principalmente en la Universidad

Nacional de Colombia sede Bogotá pero

regrese a Pasto por motivos personales, pero

pude encontrar muchas diferencias en el

transporte público de una ciudad tan grande

como lo es Bogotá y una ciudad pequeña

como lo es Pasto, observe que la ciudad al

ser pequeña presentaba problemas de caos

vehicular como los tiene Bogotá pero es

comprensible que esta ciudad los tenga ya 
que abarca gran cantidad de personas, pero Pasto no es una ciudad metropolitana lo que me hizo entender que había una falencia de diseño en la organización del transporte público así mismo de cultura ciudadana.

Kevin Alexander Montenegro Bravo, estudiante del Departamento de Diseño Industrial de la Universidad de Nariño de la ciudad de San Juan de Pasto. Nací en la ciudad de Bogotá el 15 de mayo de 1994 soy dibujante técnico y arquitectónico, inicie mis estudios de diseño en la universidad de Nariño en pasto, hasta ahora es un logro muy importante en mi vida, he vivido en pasto desde los 5 años y he visto prácticamente la evolución social cultural y estructural de toda la ciudad, por lo que me impulse al proyecto de movilidad ya que durante muchos años he acumulado experiencias referentes a este tema y me parece una gran oportunidad poder contribuirle algo a la ciudad que me ha visto crecer. 


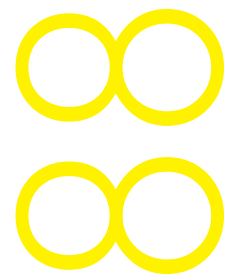

